\title{
Metastatic Colorectal Cancer Treated with FOLFOX + Cetuximab in Long Term Use Protocol. Complete Responses and Acceptable Tolerability Profile. Case Series
}

\section{Abstract}

Cetuximab, an anti-EGFR monoclonal antibody, has shown benefits when combined with the standard chemotherapy protocol for treating metastatic colorectal cancer (mCRC) in patients with wild-type Kras, either as the first-line or subsequent-line treatment. The most frequent profiles of toxicity are hematologic ( $8 \%$ grade 3 and $5 \%$ grade 4 ), dermatologic (24\% grade 3 and $2 \%$ grade 4 ), acneiform exanthem (17\% grade 3 and $0 \%$ grade 4 ) and diarrhea (10\% grade 3 and $1 \%$ grade 4), which are critical for the maintenance of therapeutic regimens until disease progression. Thus, this study aims to report the cases of two patients with $\mathrm{MCRC}$ undergoing FOLFOX protocol associated with Cetuximab as first-line for metastatic disease. Both patients were male, older and with good clinical performance (ECOG 0.1). The subjects were followed for five years. Radiological complete responses were observed, encouraging continuity of care in Long Term Use (LTU). Clinical and radiological outcomes were unequivocally represented by complete responses, associated with acceptable and manageable tolerability profiles, culminating in overall survival (OS) over 60 months (5 years) from diagnosis. Thus, the data reported in this case series corroborate the data from current studies demonstrating benefits of anti-EGFR therapy in selected populations of patients with wild-type Kras, as first shown in OPUS study and more recently in the CALGB/ SWOG 80405 study, emphasizing that treatment of mCRC with new oncogenetically guided approaches can significantly increase OS and tumor regression rates at LTU protocols.
Besteiro A.V.M.1,2,

Puty T.C.1,3

Dias M.S. 1,3,

Semblano A.A.P.1,2,

Carvalho L.E.W.1

1 Oncologica Brazil - Education and Research.

2 Centro Universitário do Estado do Pará. 3 Universidade do Estado do Pará.

\section{Contact information:}

Luis Eduardo Werneck de Carvalho.

Address: Av. Visconde de Souza Franco, 570. Zip Code 66053-000.

Tel: +559132235800.

झdreduardocarvalho@oncologica.com.br.

\footnotetext{
Keywords

Colorectal Cancer; Metastasis; Complete Radiological Response; Toxicity Management; Overall Survival.
} 


\section{Introduction}

Colorectal Cancer (CRC) is the third most common cancer type in the world [1]. Metastasis to the liver and lungs are the main causes of death, present in about $40-50 \%$ of cases [2]. One of the treatments of choice for metastatic colorectal cancer (mCRC) is defined by mFOLFOX6 Protocol (Oxaliplatin 85 $\mathrm{mg} / \mathrm{m}^{2}$ IV over 2 hours in D1, Leucovorin $400 \mathrm{mg} /$ $\mathrm{m}^{2}$ IV over 2 hours also in D1, 5-Fluorouracil (5-FU) $400 \mathrm{mg} / \mathrm{m}^{2}$ bolus followed by 5 -FU $2,400 \mathrm{mg} / \mathrm{m}^{2}$ IV continuous infusion for 46 hours in D1 every two weeks). This protocol has proven to be effective in the treatment of $\mathrm{mCRC}$ with increased OS of approximately 21 months [3].

New drugs classified as targeted therapies, such as Cetuximab (inhibitor epidermal growth factor receptor-EGFR), have a satisfactory response in combination with standard chemotherapy protocols mFOLFOX6 and FOLFIRI (Irinotecan, 5-FU, and Leucovorin) [3-9], in patients with wild-type Kras $[10,11]$. However, the control of adverse reactions, especially in the long-term, makes adherence to the associated treatment difficult (e.g. peripheral neuropathy, common side effect of Oxaliplatin, and skin rash, diarrhea, hypertension, anorexia, and mucositis/stomatitis, mainly caused by Cetuximab $[12,13])$.

Taking this into account, the assessment of the control of adverse reactions to the combined treatment of mFOLFOX6 + Cetuximab, mainly in patients receiving this medication in the long term, is highly relevant. Therefore, this work describes a series of two cases diagnosed with MCRC who presented wild-type Kras with favorable clinical performance, according to the Eastern Cooperative Oncology Group (ECOG), which were treated with a combination of mFOLFOX6 + Cetuximab in Long Term Use (LTU) maintenance scheme [14].

\section{Case 1}

Written informed consent was obtained from the patient for publication of this report and any associated images.
60 year-old male patient receiving care in the oncology service since October 2008, with diagnosis confirmed by histopathological evaluation of adenocarcinoma in the right colon, moderately differentiated and measuring $5.0 \mathrm{~cm}$. The tumor had angiolymphatic infiltration and metastasis in ten of twenty pericolonic lymph nodes (10/20) with T3N2MX staging.

Right partial colectomy was performed two months after the diagnosis. In February 2009, the patient began treatment with adjuvant FOLFOX4 protocol, in another clinic, presenting control of disease evolution. After 18 months of initial diagnosis, positron emission tomography-computed tomography (PET-CT) was performed in May 2010, for control, which showed presence of two hypermetabolic nodules in right pulmonary hilum, measuring 0.6 $\mathrm{cm}$ (SUV MAX = 1.8) and $0.9 \mathrm{~cm}$ (SUV MAX = 3.4) (Figure 1A), defined as secondary implants and raising its staging to T3N2M1, determining metastatic recurrence at the time.

In March 2011, following the interrupted protocol in the previous practice, the patient had occasional episodes of diarrhea and dyspnea on mild exertion, associated with non-productive chronic cough. Thoracoabdominal computed tomography (CT) was requested, which revealed the presence of a third node located in the lower lingular segment, measuring $0.5 \mathrm{~cm}$ - totaling three nodules in lung topography.

In November 2011, after having access to Kras testing, recently available in Brazil, the patient reinitiated rescue chemotherapy for recurrent metastatic disease in palliative regime, following eight cycles of mFOLFOX6 Protocol (Oxaliplatin $85 \mathrm{mg} / \mathrm{m}^{2}$ D1, Leucovorin $400 \mathrm{mg} / \mathrm{m}^{2}$ bolus D1 and $2400 \mathrm{mg} / \mathrm{m}^{2}$ - 46 hours continuous infusion) associated with Cetuximab (loading dose of $400 \mathrm{mg} / \mathrm{m}^{2}$ and weekly dose of $250 \mathrm{mg} / \mathrm{m}^{2}$, in the classical protocol). Initially, adverse effects were reported, such as diarrhea, asthenia and emesis, 0-1 degree, according to the National Cancer Institute Common Terminology Criteria for Adverse Events (NCI-CTCAE). 
Figure 1: (A) PET-CT showing hypermetabolic nodules in the lower lobe of the right lung (05/2010); (B) PETCT following treatment with standard procedures, showing no hypermetabolic lesions (03/2012).

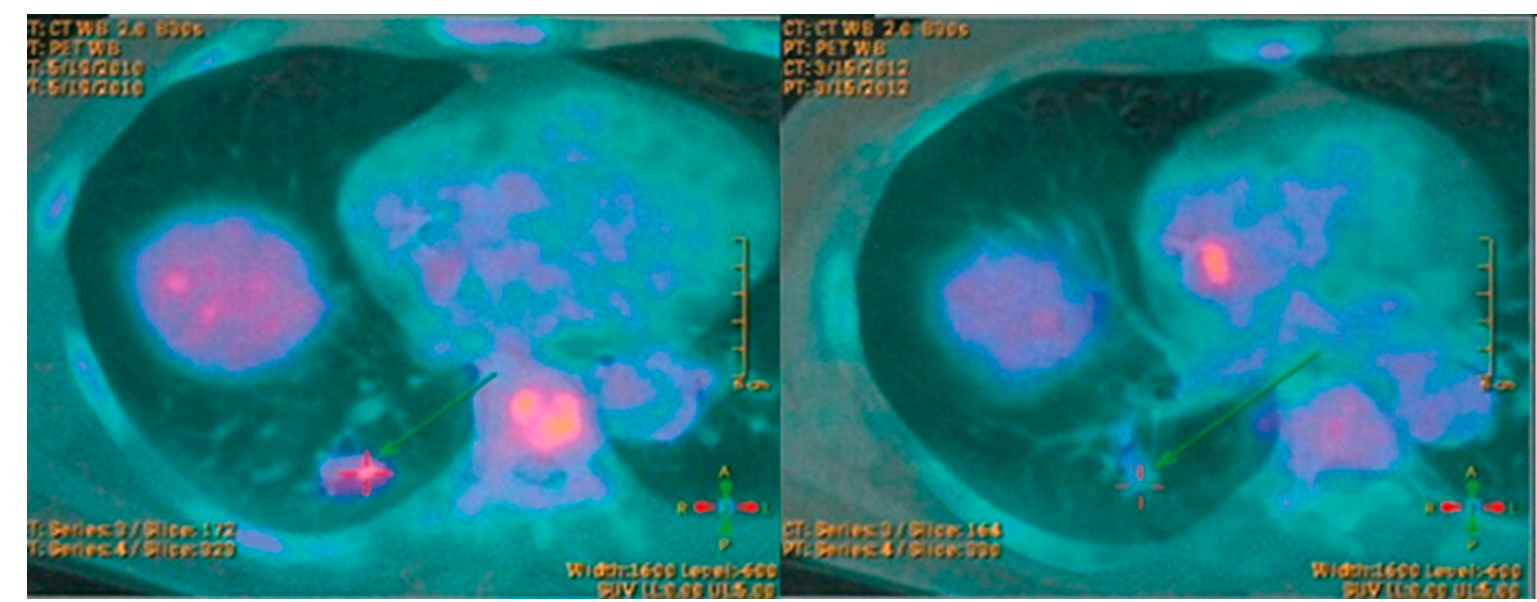

Five months after the initiation of rescue therapy with Cetuximab, new PET-CT (Figure 1B) showed complete eradication of hypermetabolic lesions in the lung. Patient remained asymptomatic, with the tumor marker carcinoembryonic antigen (CEA) stabilized at $4.25 \mathrm{ng} / \mathrm{ml}$, presenting adverse reactions grade 0-1, with a slight predominance of skin rash, which was later defined as a predictive factor of good response to anti-EGFR agent.

After completing the eighth cycle of first-line rescue treatment, maintenance treatment with Cetuximab only was established. After the third week of the beginning of Cetuximab treatment, the patient presented skin rash grade 2 . However, there was no need for dose adjustment or even for changing the proposed regimen, since all reported adverse effects were handled with preventive clinical and adequate curative protocols, with occasional use (3 times) of doxycycline $100 \mathrm{mg}$ orally for 5 days.

The patient presented significant clinical improvement, weight gain, was asymptomatic, and had complete radiological response to the PET-CT (Figure 2), with a significant fall of the CEA to $2.20 \mathrm{ng} / \mathrm{ml}$.

After 80 months of diagnosis and 44 months of use of anti-EGFR therapy, the patient continues in the maintenance scheme with isolated Cetuximab in intervals of 15 days (modification of protocol to biweekly dose of $500 \mathrm{mg} / \mathrm{m}^{2}$ ). Patient is asympto-
Figure 2: Radiological control PET-CT after 80 months of diagnosis showing no hypermetabolic lesions and complete and sustained radiological response to treatment.

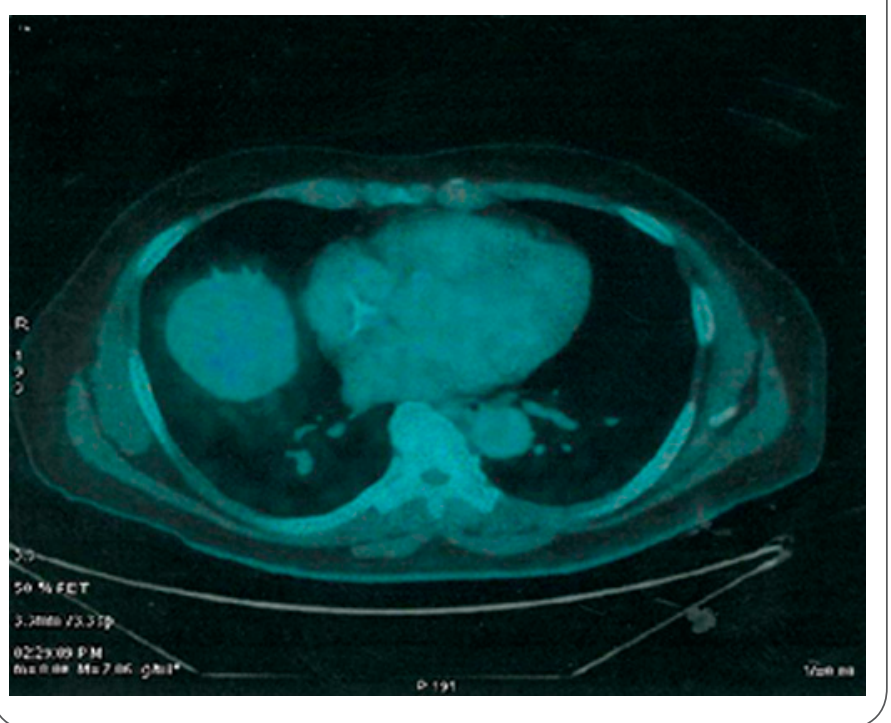

matic, with toxicities grade 0-1 and CEA $2.93 \mathrm{ng} /$ $\mathrm{ml}$, with no evidence of clinical, radiological or laboratory progression.

\section{Case 2}

Written informed consent was obtained from the patient for publication of this report and any associated images. 
Figure 3: Evolution of CEA tumor marker in the patient during treatment.

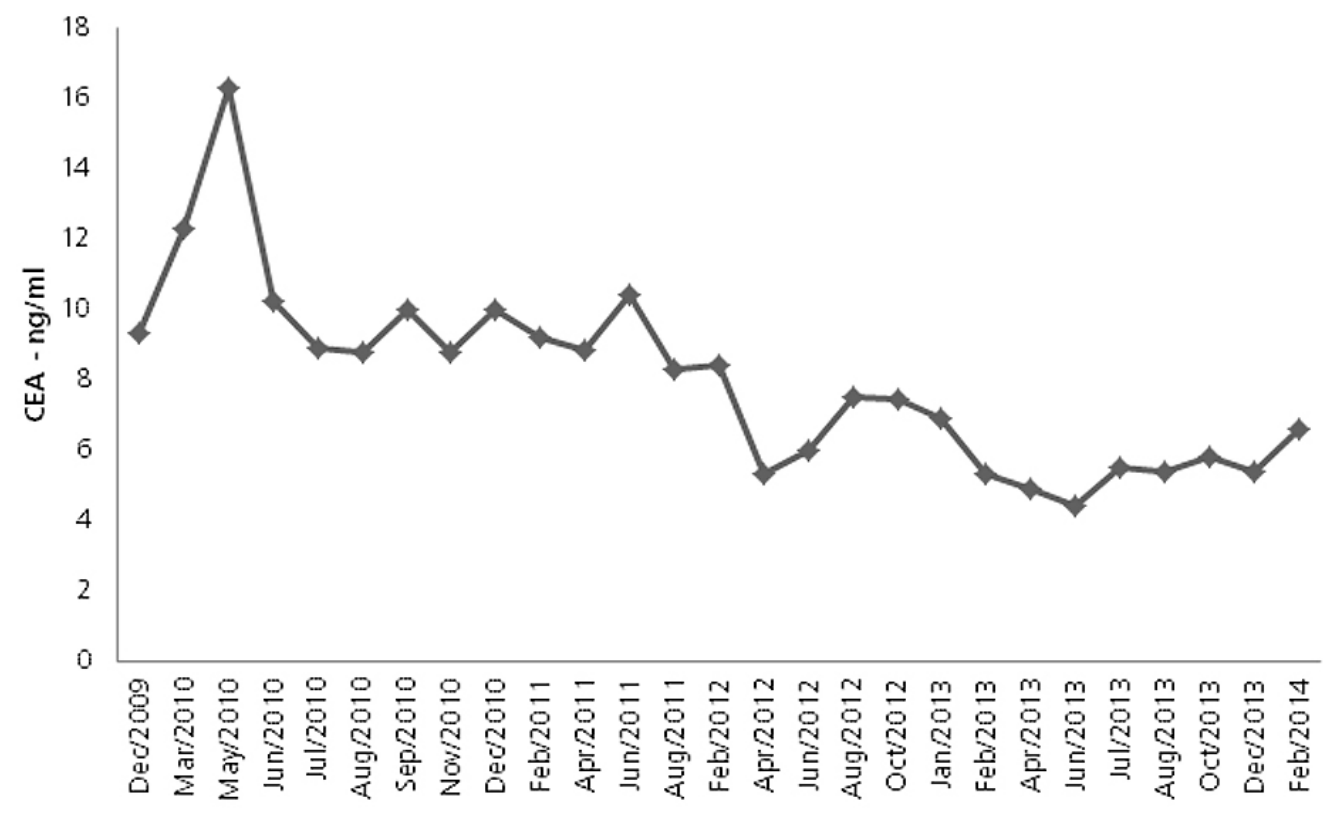

80 year-old male patient, receiving care in the oncology service since April 2009, had ulcerated lesion in the colon, as assessed by colonoscopy. By histopathological exams, a moderately differentiated ulcerative-infiltrative adenocarcinoma was diagnosed, located in the ascending colon, with angiolymphatic invasion and metastasis in two out of nine pericolonic lymph nodes (2/9). Patient was staged as T3N1MX at the time of initial diagnosis.

Two months after diagnosis, hemicolectomy was performed, followed by adjuvant chemotherapy with mFOLFOX6 protocol for 12 cycles, with subsequent control of evolution. In March 2010, there was an increase of the tumor marker CEA from $9.31 \mathrm{ng} / \mathrm{ml}$ to $12.28 \mathrm{ng} / \mathrm{ml}$. Genetic assessment of Kras was requested, which showed the patient had the wild-type.

At the end of the 8th cycle with mFOLFOX6, in April 2010, treatment with Cetuximab was initiated $\left(400 \mathrm{mg} / \mathrm{m}^{2}\right.$ loading dose and subsequent dose of $250 \mathrm{mg} / \mathrm{m}^{2}$ weekly), because patient presented progression of CEA tumor to $16.38 \mathrm{ng} / \mathrm{ml}$ (Figure 3), in addition to presenting the wild-type Kras in metastatic disease of lymph nodes.
Figure 4: Computed tomography (CT) of the chest showing no pulmonary micronodules in lower lobe of the right lung.

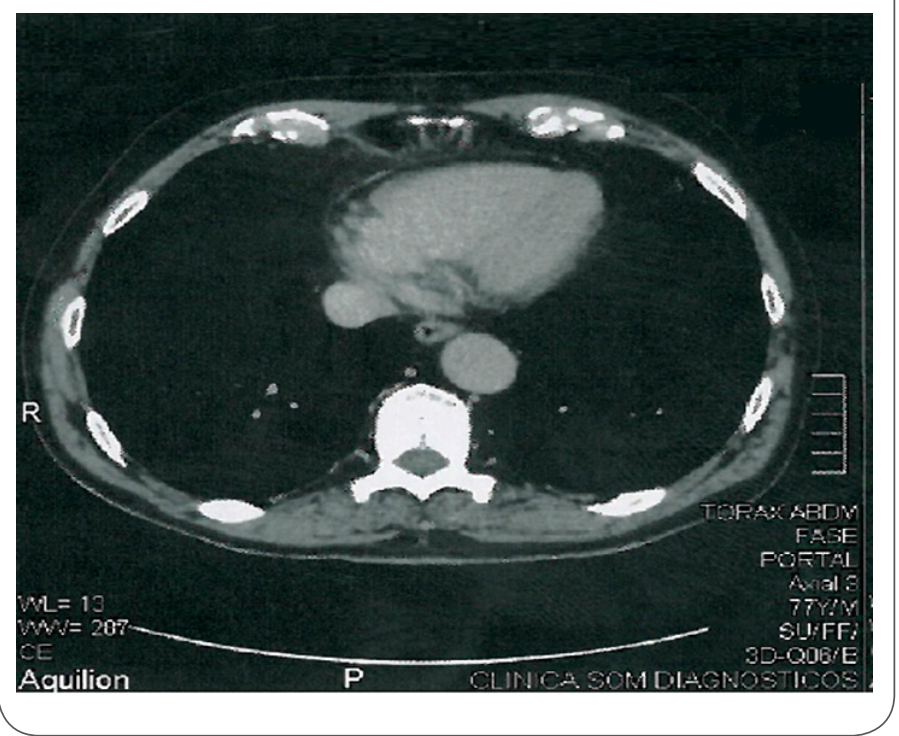

After the fourth month of therapy with Cetuximab + mFOLFOX6, Cetuximab only protocol was used until progression or toxicity. Worsening of nail lesions was reported after the first cycle with antiEGFR therapy alone, requiring the use of doxycycline (100 mg PO D1 to D5), without the need of topical corticosteroid therapy or standard protocol dose adjustment. 
In June 2012, CT revealed two micronodules of $0.4 \mathrm{~cm}$ in right lung lower lobe not considered as disease progression. Predicted therapy with Cetuximab for 36 months was maintained, occurring subsequent reduction of CEA to $5.95 \mathrm{ng} / \mathrm{ml}$. Three months later, new CT (Figure 4) showed complete radiological response with no evidence of metastasis in the lungs or elsewhere.

After 72 months of diagnosis, with 36 cycles carried out in LTU scheme with Cetuximab only, the patient was asymptomatic, with no notable toxicities, CEA stabilized at $5.3 \mathrm{ng} / \mathrm{ml}$ and no evidence of clinical, radiological or laboratory progression. By the end of the realization of this study, the patient showed OS of 60 months from diagnosis.

\section{Discussion}

Due to the insidious onset of primary and other lesions, the diagnosis of $\mathrm{mCRC}$ is usually delayed. Even with improved OS of patients treated with mFOLFOX6, colorectal cancer stage IV still has a poor prognosis. However, anti-EGFR therapy drugs, such as Cetuximab -monoclonal antibody that binds to the extracellular portion of the receptor for the epidermal growth factor (EGFR)-, have shown very satisfactory results as a first-line associated therapy, as well as isolated maintenance therapy $[5,8,9]$.

The phase III CALGB/SWOG 80405 study, which included 1137 patients with wild-type Kras tumors initially treated with mFOLFOX6 or FOLFIRI, and then randomly assigned to receive associated Cetuximab $\left(400 \mathrm{mg} / \mathrm{m}^{2} \times 1\right.$, then $250 \mathrm{mg} / \mathrm{m}^{2}$, once a week), bevacizumab (5 mg/kg twice a week), or both. The median OS for mFOLFOX6 associated with Cetuximab was 30.1 months compared with 26.9 months when mFOLFOX6 was associated with bevacizumab [14].

In this series of cases, the patients were submitted to mFOLFOX6 protocol for 8 cycles, followed by maintenance with Cetuximab until progression or toxicity $[3-5,7,9,14]$. Both responded with comple- te and continuous response of the disease for over 60 months. The tests of follow-up and evolution control, such as PET-CT, chest, abdomen and pelvis $\mathrm{CT}$ and colonoscopy showed complete radiological response with ETS (Early Tumor Shrinkage) in lung metastases after 2 months [15], in the first case. In summary, patients did not show metastatic disease anymore.

The toxicity profile of grade 3 and 4 patients treated with Cetuximab is composed mainly by hematotoxicity (21\%-24\%), skin reactions (24\%-22\%), acneiform exanthem/rash (17\%-20\%) and diarrhea $(10 \%-11 \%)[6,7]$. Our patients had early skin and nail rash, in agreement with the scientific literature. In addition, this side effect may be related to the objective response, considered as a predictive factor of the response; no other alteration was reported in the subsequent sessions, even receiving treatment in LTU for more than four years in both cases [13]. Furthermore, there was no need to adjust or reduce treatment dose.

These results show the benefits of anti-EGFR therapy in selected populations of patients with wildtype Kras, as shown in the CALGB/SWOG 80405 study, emphasizing that treatment of $\mathrm{mCRC}$ with the new targeted therapies can significantly increase OS rates and tumor regression rates in LTU schemes, with manageable toxicity to treatment, even for long periods of clinical monitoring [15].

\section{Conclusion}

The reported data show overall survival far above presented by the literature, with stabilization of the CEA tumor marker, complete radiological response to PET-CT of secondary pulmonary lesions and manageable tolerability profile with the use of $\mathrm{mFOL}$ FOX6 protocol + Cetuximab and later Cetuximab only. This suggests that the maintenance protocol in LTU with Cetuximab only is an acceptable therapy for patients with $\mathrm{mCRC}$. 


\section{Acknowledgments}

We are grateful for the attention and availability of the patients described in this case report.

\section{Funding}

The group was supported by the Oncologica Brazil Education and Research, which provided us with the space, material and financial support necessary to produce this article.

\section{Conflict of Interest Disclosures}

The authors declare no conflicts of interest regarding this case report.

\section{Abbreviations}

- CRC: colorectal cancer;

- mCRC: metastatic colorectal cancer;

- EGFR: epidermal growth factor receptor;

- ECOG: Eastern Cooperative Oncology Group,

- LTU: long term use; KRAS: Kirsten rat sarcoma viral oncogene homolog;

- ETS: early tumor shrinkage; OS: overall survival;

- PET-CT: positron emission tomography-computed tomography;

- CT: computed tomography;

- CEA: carcinoembryonic antigen.

\section{References}

1. Rebecca Siegel MPH, Jiemin Ma PhD, Zhaohui Zou MS and Ahmedin Jemal DVM, PhD. Global cancer statistics. CA: A Cancer Journal for Clinicians, Volume 64, Issue 1, pages 9-29, January/February 2014

2. Woo-Jeong Jeong, Pu-Hyeon Cha, and Kang-Yell Choi. Strategies to overcome resistance to epidermal growth factor receptor monoclonal antibody therapy in metastatic colorectal cancer. World J Gastroenterol. 2014; Aug 7; 20(29): 9862-9871.

3. Tournigand C, André T, Achille E, Lledo G, Flesh M, MeryMignard D, Quinaux E, Couteau C, Buyse M, Ganem G, Landi B, Colin P, Louvet C, de Gramont A. FOLFIRI followed by FOLFOX6 or the reverse sequence in advanced colorectal cancer: a randomized GERCOR study. J Clin Oncol. 2004 Jan 15; 22(2): 229-37. Epub 2003 Dec 2.

4. Goldberg, R, Sargent DJ, Morton RF, Fuchs CS, Ramanathan RK, Williamson SK, et al. Modified FOLFOX 6 widely used but not published. J Clin Oncol 2004; 22: 23-30.

5. Bokemeyer C, Bondarenko I, Makhson A, Hartmann JT, Aparicio J, de Braud F, Donea S, Ludwig H, Schuch G, Stroh C, Loos AH, Zubel A, Koralewski P. Fluorouracil, leucovorin, and oxaliplatin with and without cetuximab in the first-line treatment of metastatic colorectal cancer. JClinOncol2009;: 27: 663-671 [PMID: 19114683 DOI: 10.1200/ jco.2008.20.8397].

6. Heinemann, V, von Weikersthal, LF, Decker, T et al. FOLFIRI plus cetuximabe versus FOLFIRI plus bevacizumab as first-line treatment for patients with metastatic colorectal cancer (FIRE3): a randomised, open-label, phase 3 trial. Lancet Oncol. 2014;: 15: 1065-107.

7. Venook, AP, Niedzwiecki, D, Lenz, $\mathrm{H}-\mathrm{J}$ et al. Phase III trial of irinotecan/5-FU/leucovorin (FOLFIRI) or oxaliplatin/5-FU/ leucovorin (mFOLFOX6) with bevacizumab (BV) or cetuximabe (CET) for patients (pts) with KRAS wild-type (wt) untreated metastatic adenocarcinoma of the colon or rectum (MCRC). Proc Am Soc Clin Oncol. 2014;: 32 (abstr LBA3.).

8. Bokemeyer C, Bondarenko I, Hartmann JT, et al: KRAS status and efficacy of first-line treatment of patients with metastatic colorectal (metastatic CRC) with FOLFOX with or without cetuximab: The OPUS experience. J Clin Oncol 26: 178s, 2008 (suppl;: abstr 4000).

9. Jonker DJ, O'Callaghan CJ, Karapetis CS, Zalcberg JR, Tu D, Au HJ, Berry SR, Krahn M, Price T, Simes RJ, Tebbutt NC, van Hazel G, Wierzbicki R, Langer C, Moore MJ. Cetuximab for the treatment of colorectal cancer. N Engl J Med. 2007 Nov 15; 357(20): 2040-8.

10. De Roock W, Piessevaux H, De Schutter J, et al: KRAS wild-type state predicts survival and is associated to early radiological response in metastatic colorectal cancer treated with cetuximab. Ann Oncol 19: 508-515, 2008. 
11. Allegra CJ, Jessup JM, Somerfield MR, et al, "American Society of Clinical Oncology Provisional Clinical Opinion: Testing for KRAS Gene Mutations in Patients With Metastatic Colorectal Carcinoma to Predict Response to Anti-Epidermal Growth Factor Receptor Monoclonal Antibody Therapy, " J Clin Oncol, 2009, 27(12): 2091-6.

12. Zhong-chuan Lv \& Jin-yao Ning \& Hong-bing Chen. Efficacy and toxicity of adding cetuximab to chemotherapy in the treatment of metastatic colorectal cancer: a meta-analysis from 12 randomized controlled trials. Tumor Biol. (2014) 35: 1174111750.

13. F. Petrelli, K. Borgonovo, S. Barni. The predictive role of skin rash with cetuximab and panitumumab in colorectal cancer patients: a systematic review and meta-analysis of published trials. Targeted Oncology. September 2013, Volume 8, Issue 3, pp 173-18.

14. Lenz H, Niedzwiecki D, Innocenti F, Blanke C, Mahony $M$, O'Neil B, Shaw J, Polite B, Hochster H, A tkins J, Goldberg R, Mayer R, Schilsky R, Bertagnolli M, Venook A. CALGB/SWOG 80405: PHASE III trial of irinotecan/5-FU/leucovorin (FOLFIRI) or oxaliplatin/5-FU/leucovorin (mFOLFOX6) with bevacizumab (BV) or cetuximab (CET) for patients (pts) with expanded ras analyses untreated metastatic adenocarcinoma of the colon or rectum. ESMO2014;: abstr 5010.

15. Susan Mayor PhD. Medical News Today. Early Tumor Shrinkage With Cetuximab Correlates With Prolonged Survival In Metastatic Colorectal Cancer. 11 June 2012 Disponível em: <http://www.medicalnewstoday.com/articles/246403.php> Acesso em: 17 abril 2015.

\section{Comment on this article:}
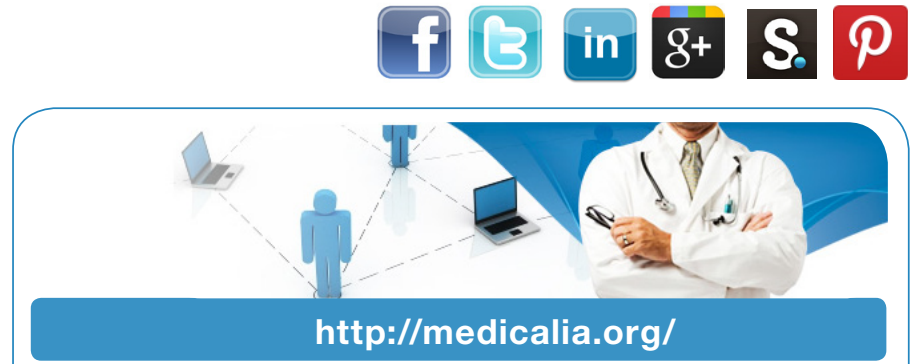

Where Doctors exchange clinical experiences, review their cases and share clinical knowledge. You can also access lots of medical publications for free. Join Now!

\section{Publish with iMedPub}

http://www.imed.pub

International Archives of Medicine is an open access journal publishing articles encompassing all aspects of medical science and clinical practice. IAM is considered a megajournal with independent sections on all areas of medicine. IAM is a really international journal with authors and board members from all around the world. The journal is widely indexed and classified Q1 in category Medicine. 